\title{
Propagating wave in active region-loops, located over the solar disk observed by the Interface Region Imaging Spectrograph ${ }^{\star}$
}

\author{
B. Zhang ${ }^{1,2}$, Y. J. Hou ${ }^{1,2}$, and J. Zhang ${ }^{1,2}$ \\ ${ }^{1}$ CAS Key Laboratory of Solar Activity, National Astronomical Observatories, Chinese Academy of Sciences, Beijing 100012 , \\ PR China \\ e-mail: yijunhou@nao.cas.cn, zjun@nao.cas.cn \\ 2 School of Astronomy and Space Science, University of Chinese Academy of Sciences, Beijing 100049, PR China
}

Received 25 April 2017 / Accepted 4 January 2018

\begin{abstract}
Aims. We aim to ascertain the physical parameters of a propagating wave over the solar disk detected by the Interface Region Imaging Spectrograph (IRIS).

Methods. Using imaging data from the IRIS and the Solar Dynamic Observatory (SDO), we tracked bright spots to determine the parameters of a propagating transverse wave in active region (AR) loops triggered by activation of a filament. Deriving the Doppler velocity of Si IV line from spectral observations of IRIS, we have determined the rotating directions of active region loops which are relevant to the wave.

Results. On 2015 December 19, a filament was located on the polarity inversion line of the NOAA AR 12470. The filament was activated and then caused a C1.1 two-ribbon flare. Between the flare ribbons, two rotation motions of a set of bright loops were observed to appear in turn with opposite directions. Following the end of the second rotation, a propagating wave and an associated transverse oscillation were detected in these bright loops. In $1400 \AA$ channel, there was bright material flowing along the loops in a wave-like manner, with a period of $\sim 128 \mathrm{~s}$ and a mean amplitude of $\sim 880 \mathrm{~km}$. For the transverse oscillation, we tracked a given loop and determine the transverse positions of the tracking loop in a limited longitudinal range. In both of $1400 \AA$ and $171 \AA$ channels, approximately four periods are distinguished during the transverse oscillation. The mean period of the oscillation is estimated as $\sim 143 \mathrm{~s}$ and the displacement amplitude as between $\sim 1370 \mathrm{~km}$ and $\sim 690 \mathrm{~km}$. We interpret these oscillations as a propagating kink wave and obtain its speed of $\sim 1400 \mathrm{~km} \mathrm{~s}^{-1}$.

Conclusions. Our observations reveal that a flare associated with filament activation could trigger a kink propagating wave in active region loops over the solar disk.
\end{abstract}

Key words. sunspots - Sun: atmosphere - Sun: filaments, prominences - Sun: oscillations

\section{Introduction}

The solar corona consists of ionized plasma with a temperature of millions of kelvins, about three orders of magnitude higher than the temperature of the visible solar surface (Erdélyi \& Fedun 2007). The heating process that generates and sustains the hot corona is still an unresolved problem in solar physics. It is believed that the subphotospheric convection zone supplies enough kinetic energy to the corona, where sufficient energy may be dissipated resulting in the heating of coronal plasma. Parker (1988) put forward that the magnetic reconnection of neighboring stressed field lines with opposite polarity vector components would release magnetic energy violently and contributes to heating the corona. This idea was followed by a large number of studies (Solanki et al. 2003; Klimchuk 2006; Bradshaw \& Klimchuk 2015; Wright et al. 2017). A possible scenario of energy transporting from the convection zone to the corona is that the convection below the solar visible surface and solar global oscillations may produce magnetohydrodynamic (MHD) waves in the photosphere, which then propagate upward into the corona carrying energy (Alfvén 1947; Parker 1958; De Pontieu

\footnotetext{
${ }^{\star}$ Movies associated to Figs. $1-4$ are available at https: //www . aanda.org
}

et al. 2007; Tomczyk et al. 2007; Antolin et al. 2017). In uniform plasmas, there are three basic types of MHD waves: slow and fast magnetoacoustic waves, and Alfvén waves. According to the frequencies observed by solar instruments, MHD modes in cylindrical plasma structures could be mainly grouped into four categories: kink, sausage, longitudinal, and torsional (Van Doorsselaere et al. 2008a). The first three modes are magnetoacoustic and hence compressible while the last one is the incompressible Alfvén mode.

With the progress in the spatial and temporary resolution of solar observation instruments, abundant evidence of various types of MHD waves (standing and propagating waves) have been detected in various solar activities. Propagating and standing transverse waves have been observed in spicules (He et al. 2009; Zaqarashvili et al. 2007; Okamoto \& De Pontieu 2011; Pereira et al. 2016), in active region (AR) fibrils (Pietarila et al. 2011; Jafarzadeh et al. 2017), in mottles (Kuridze et al. 2012, 2013), in filament threads (Lin et al. 2007; Terradas et al. 2008; Li \& Zhang 2012; Shen et al. 2014) and in coronal loops (Ofman \& Wang 2008; Tian et al. 2014; Guo et al. 2015; Li et al. 2017). Most of these events could be explained as the magnetic flux tubes being perturbed by an external excitation, and this perturbation appears as transverse oscillation and propagates along the 

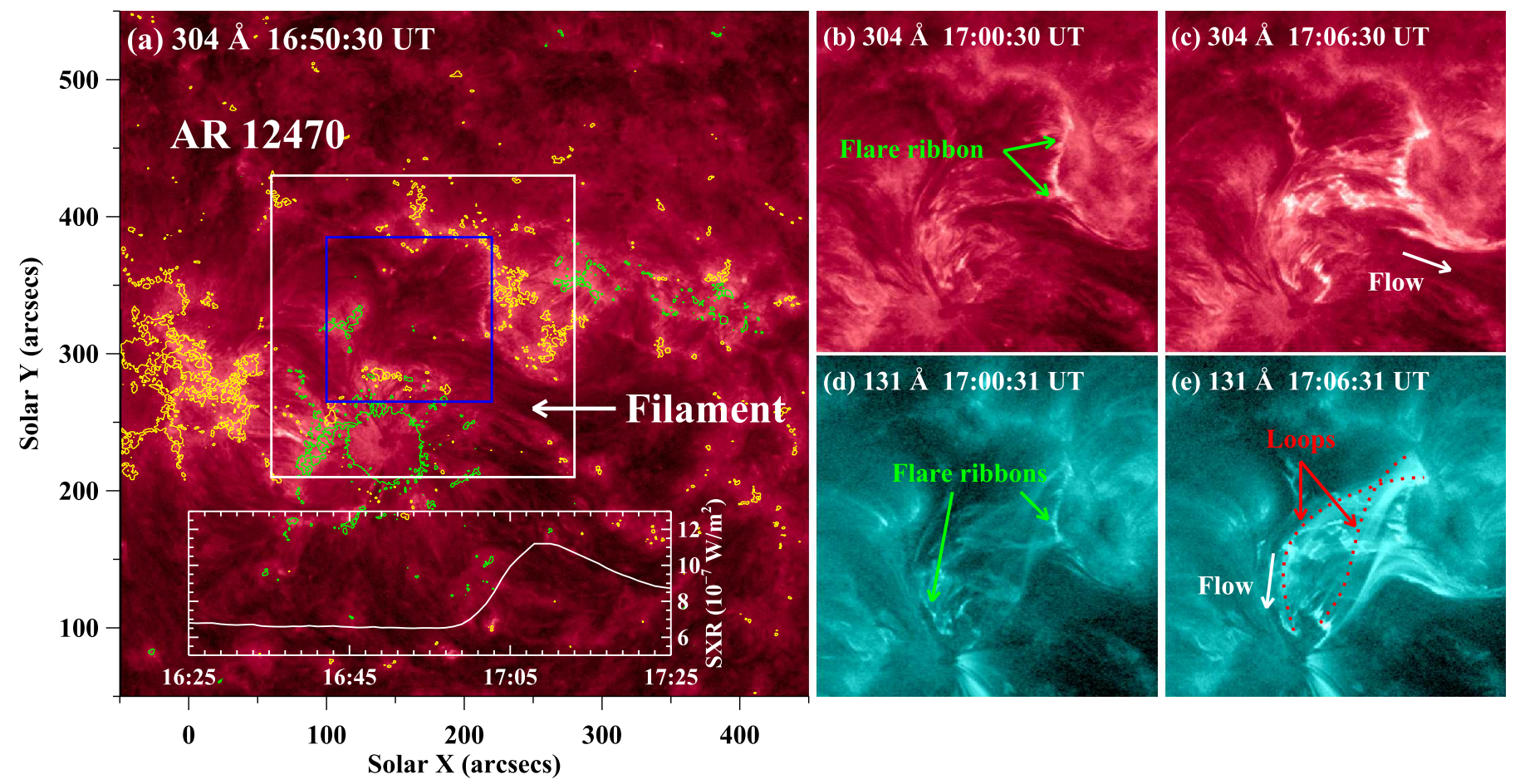

Fig. 1. Panel a: SDO/AIA $304 \AA$ Amage displaying the overview of the NOAA AR 12470 on 2015 December 19 . The filament before activation is denoted by the white arrow. The white square outlines the field of view (FOV) of panels $b-e$ while the blue square represents the FOV of $1400 \AA$ images in Fig. 2. The green and yellow curves are contours of corresponding negative and positive magnetic fields, respectively. The white curve in the bottom shows GOES SXR 1-8 Å flux of the associated C1.1 flare. Panels $b-e$ : sequence of AIA $304 \AA$ and $131 \AA$ images showing the flare caused by the filament's activation in two different temperatures. The green arrows in panels $b$ and $d$ mark the flare ribbons while the white arrows in panels $c$ and $e$ approximate the trajectory of the filament material flows. The red dotted lines in panel $e$ indicate the flare loops with shear in high temperature. An animation of the $131 \AA$ and $304 \AA$ images is available online.

flux tube, which is usually detected as a temporal variation of the magnetic tube position or the Doppler velocity (Oliver et al. 2014).

In recent years, the new-generation satellites such as Solar Dynamic Observatory (SDO; Pesnell et al. 2012) and Interface Region Imaging Spectrograph (IRIS; De Pontieu et al. 2014) have provided observations that have high tempo-spatial resolution ranging from the photosphere, chromosphere, and transition region to corona. Although there have been many studies of MHD waves in the last few decades, observations about the propagating wave taken by the IRIS are rarely reported (Okamoto et al. 2015). Over the solar disk, there are only a few published results of chromospheric waves from the IRIS data (Bryans et al. 2016; Kanoh et al. 2016). One important reason is that there are so many fine structures in the chromosphere, thus making it difficult to detect the wave signal in a particular chromospheric structure among the abundant features. By checking 2 yr of IRIS data (from June 2014 to May 2016), we find an example of a wave-oscillation event over the disk on 2015 December 19. This event enables us to examine the photospheric magnetic field environment. In this paper, we report on the waveoscillation event in active region loops triggered by filament activation which results in the storing and releasing of twist in the loops.

The remainder of this paper is structured as follows. Sect. 2 contains the observations and data analysis taken in our study. The observations of the filament activation, flare eruption, rotation motions, and the subsequent propagating transverse wave in the loops are presented in Sect. 3. Finally, in Sect. 4 we conclude this work and discuss the results.

\section{Observations and data analysis}

On 2015 December 19, the IRIS was pointed at NOAA AR 12470 from 16:41:13 UT to 17:37:33 UT, with the central coordinate of $\left(152^{\prime \prime}, 302^{\prime \prime}\right)$. We hence obtained a series of slit-jaw $1400 \AA$ images (SJIs) with a cadence of $13 \mathrm{~s}$, a pixel scale of $0 . .^{\prime \prime} 333$, and a field of view (FOV) of $167^{\prime \prime} \times 175^{\prime \prime}$. The $1400 \AA$ channel contains emission from the Si IV 1394/1403 $\AA$ lines that are formed in the lower transition region, which include the contributions from continuum that is formed in lower chromosphere as well. The employed data are all level 2, which have applied dark current subtraction, flat field, geometrical, and orbital variation corrections (De Pontieu et al. 2014). For the spectroscopic analysis, we mainly adopted the Si IV $1402.77 \AA$ line which is formed in the middle transition region with a temperature of about $10^{4.9}$ $\mathrm{K}$ (Li et al. 2014; Tian et al. 2014). The spectral data were taken in a large sit-and-stare mode with a step cadence of $3.2 \mathrm{~s}$. As the Si IV profiles were relatively close to a single Gaussian, we used single-Gaussian fits to approximate the $1402.77 \AA$ line (Peter et al. 2014).

The Atmospheric Imaging Assembly (AIA; Lemen et al. 2012) and the Helioseismic and Magnetic Imager (HMI; Schou et al. 2012) observations from the SDO were also used. The AIA continuously observes the multilayered solar atmosphere, including the photosphere, chromosphere, transition region, and corona, in ten (E)UV passbands with a cadence of $12 \mathrm{~s}$ and a spatial resolution of $1 . .^{\prime \prime} 2$. The observations of $304 \AA, 171 \AA$, and $131 \AA$ on 2015 December 19 were employed in the present work. We also applied the corresponding full-disk line of sight (LOS) magnetograms from the HMI, with a cadence of $45 \mathrm{~s}$ and 

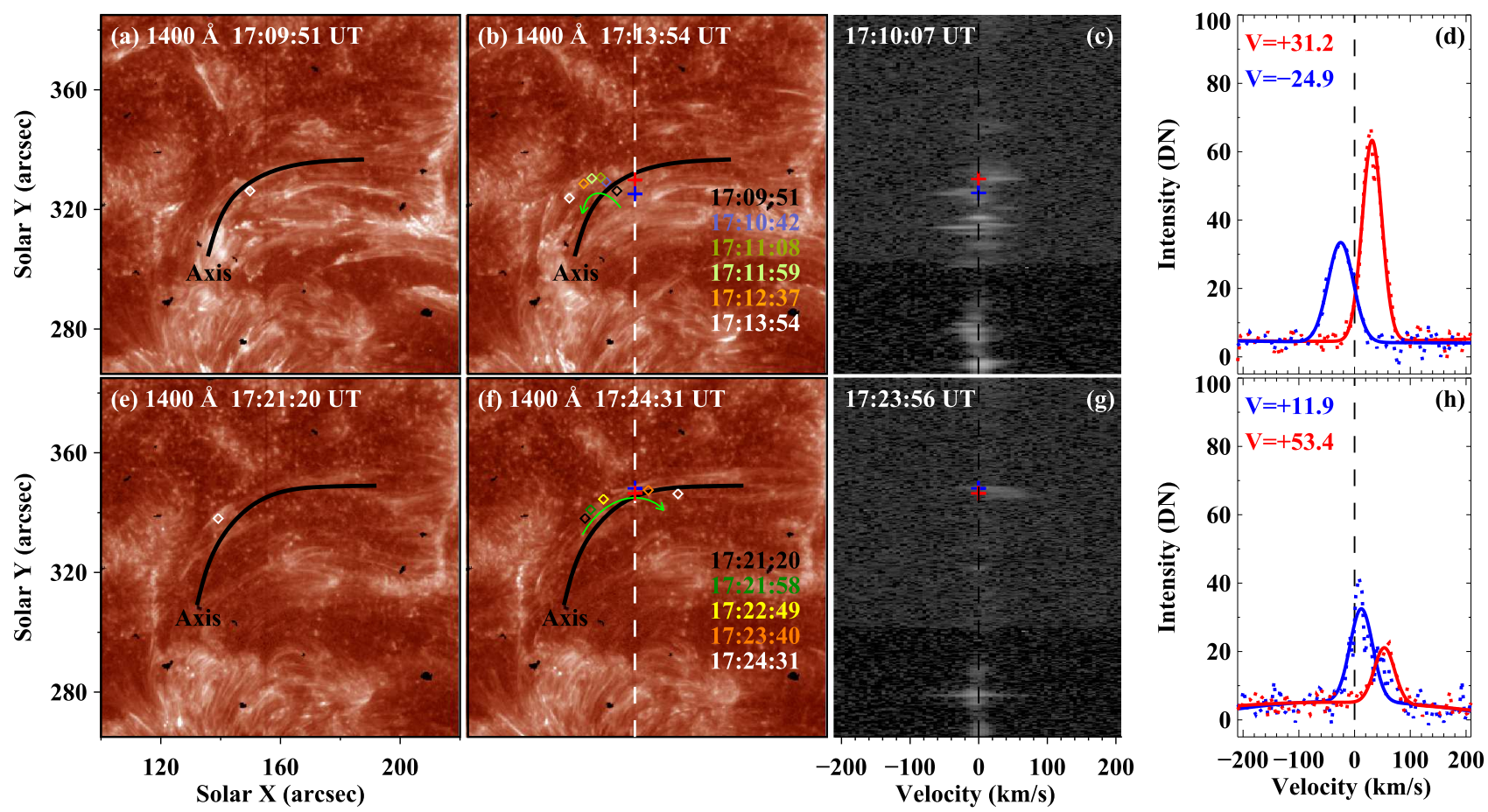

Fig. 2. Panels $a-b$ : IRIS SJIs of $1400 \AA$ Aisplaying the first rotation motion around 17:10 UT on 2015 December 19. The black line approximates the axis of the rotation while the green arrow marks the direction of the rotation. The positions of the tracked feature in different times are denoted by diamonds of different colors, of which the white one represents the current position. The white dashed line in panel $b$ shows the location of slit. Panels $c-d$ : Si IV $1402.77 \AA$ line spectra along the slit in panel $b$ and the profiles (dotted lines) and its Gaussian fittings (solid lines) of this line at blue and red plus positions in panels $b$ and $c$. The observation time is at 17:10:07 UT. Panels $e-f$ : IRIS 1400 $\AA$ images exhibiting the second rotation motion of the loops just before the appearance of a propagating wave. The features marked here are similar to those in panels $a-b$. Panels $g-h$ : similar to panels $c-d$, but at 17:23:56 UT. The temporal evolution of the two rotation motions is available as an online animation.

a pixel size of $0 . " 5$. Using the standard routine "hmi_prep" in Solar Software package, we calibrated the AIA and HMI data to a specified pointing, rescaled the images to a spatial resolution of $1 . .^{\prime \prime} 2$, and de-rotated these images (removing the roll angle) so that the solar east-west and north-south axes were aligned with the horizontal and vertical axes of the image, respectively. Then these data were all de-rotated differently to a reference time (16:41:13 UT, the start time of the IRIS observations). The IRIS observations were also all de-rotated to the same time. Finally, we aligned the IRIS data with the SDO observations according to specific features such as bright spots and loops which were observed by both the IRIS and SDO.

\section{Results}

\subsection{Activation of a filament and onset of the subsequent flare}

On 2015 December 19, NOAA AR 12470 was located near the solar disk-center. Around the main sunspot of this AR, a C1.1 flare with two ribbons took place near 17:00 UT, which was caused by a filament's activation (see animation). Plenty of filament material was brightened and then displayed different dynamic evolutions. Partial bright material moved along the filament's axis and slipped away. However, some other bright material interacted with the active region loops which overlaid the filament. These loops were disturbed by the filament material and underwent twice rotating motions from 17:09 UT to 17:26 UT. Subsequently, a propagating wave and the associated transverse oscillation were detected. Figure 1 shows the overview of the area of interest, the process of the filament's activation, and the flare's onset in AIA $304 \AA$ and $131 \AA$ channels. The AR's main sunspot with negative polarity was surrounded by positive fields. And a filament lied on the polarity inversion line (PIL) of this AR (see panel (a)). The C1.1 flare started at 16:58 UT, and at the onset of the flare, the brightening of the western footpoints of the flare loops gradually propagated toward the southwest (see panel (b)), which developed into the western flare ribbon above positive magnetic fields. The eastern flare ribbon of this flare was located in the sunspot umbra with strong negative field (panel (d)). Between these two ribbons, the flare loops with evident shear appeared in $131 \AA$ channel (see panel (e)), which is dominated by the emission from Fe XXI line [log $T \sim 7.05]$ (O'Dwyer et al. 2010). Meanwhile, the activated filament material was detected to flow from the north-east to the south-west with a projected speed of $\sim 90 \mathrm{~km} \mathrm{~s}^{-1}$ (see panel (c)). Another flow was observed along the flare loops, which fell down in the sunspot umbra (see panel (e)). This flare increased to its maximum at 17:10 UT and faded away in the following ten minutes (see the GOES flux curve in the bottom of panel (a)).

\subsection{Rotations of active region loops}

Following the flare loops' appearance, two rotation motions were observed in the loops connecting negative-polarity sunspot with northwestern positive fields (see animation). Around 17:09 UT, the peak of the flare, a set of bright loops appeared in $1400 \AA$ images (see Fig. 2a). Then the first rotation motion took place during these loops, lasting from 17:09 UT to 17:15 UT. Examining the AIA $304 \AA$ and $131 \AA$ observations (see animation), we noticed that some bright material which came from the activated filament moved in the rotating loops. We chose one 
bright spot which is relatively isolated in $1400 \AA$ channel and determine the positions of the tracked spot at different times (see panel (b)). Looking from left to right along the rotation axis, the bright spot rotated about the axis anticlockwise. To confirm this rotation motion, we employ the IRIS spectra data for Doppler velocity measurement. Panel (c) shows the Si IV $1402.77 \AA$ line spectra along the slit. The positions at which the rotating loops cross the slit are indicated by red and blue plus symbols in the figure. The spectra profiles and Gaussian fits of these two positions are shown in panel (d). Here we get the Gaussian fit to the Si IV profiles by computing a non-linear least-squares fit to function $f(x)$ with six parameters. The function $f(x)$ is a linear combination of a Gaussian and a quadratic: $f(x)=A_{0} \mathrm{e}^{\frac{-z^{2}}{2}}+A_{3}+A_{4} x+A_{5} x^{2}$, where $z=\frac{x-A_{1}}{A_{2}}$. The six parameters represent the height of the Gaussian $\left(A_{0}\right)$, the center of the Gaussian $\left(A_{1}\right)$, the width of the Gaussian $\left(A_{2}\right)$, the constant term $\left(A_{3}\right)$, the linear term $\left(A_{4}\right)$, and the quadratic term $\left(A_{5}\right)$, respectively. Meanwhile, one-sigma error estimates of the six returned parameters are also given. As a result, we calculate the uncertainty of the Doppler velocity according to the one-sigma error estimate of the center of the Gaussian $\left(A_{1}\right)(\mathrm{Li}$ et al. 2016). At 17:10:07 UT, the Doppler velocities at blue and red plus positions are respectively $-24.9 \pm 0.9 \mathrm{~km} \mathrm{~s}^{-1}$ (blueshift) and $31.2 \pm 0.5 \mathrm{~km} \mathrm{~s}^{-1}$ (redshift). The pair of blueshift and redshift signals provide a strong evidence for the rotation motion. Undergoing this rotation, a set of loops moved northward and reached the northernmost point around 17:21 UT. Then, from 17:21 UT to 17:24 UT, the second rotation motion of these loops was detected again. But this time, the rotation was clockwise if one looks from left to right along the rotation shaft (see panel (f)). The spectral data at 17:23:56 UT were taken to investigate the second rotation motion. Through similar analysis method mentioned above, we estimate the Doppler velocities at the positions indicated in the figure as $11.9 \pm 1.8 \mathrm{~km} \mathrm{~s}^{-1}$ and $53.4 \pm 1.5 \mathrm{~km} \mathrm{~s}^{-1}$ (panel (h)), respectively.

\subsection{A propagating wave with transverse oscillation in the loops}

The loops undergoing two rotation motions were clearly observed in $171 \AA$ passband too (see Fig. 3a). Comparing the $171 \AA$ images with HMI magnetograms, we can determine that the loops originated from the main sunspot with negative polarity and connected to the northwestern region with positive fields (see panel (b)). At the later phase of the second rotation, several bright spots moved in a wave-like manner from left to right along the active region loops. We chose one distinct bright spot (with the maximum brightness within the target region) at 17:24:57 UT in $1400 \AA$ channel. We tracked this spot and determined the coordinates of the spot frame-by-frame from 17:24:57 UT to 17:32:23 UT (see animation). After repeating the tracking process for ten times, we obtained ten groups of the spot's coordinates at each frame. Then, we got the average values of the spot's coordinates at each frame and took the standard deviation as the uncertainty. At about 17:25 UT, this bright spot moved along these loops toward the west with a mean projected velocity of $66.2 \pm 1.1 \mathrm{~km} \mathrm{~s}^{-1}$ (see panel (c)) and moved back to the east after 17:29 UT with an average projected velocity of $12.7 \pm 1.3 \mathrm{~km} \mathrm{~s}^{-1}$ (see panel (d)). The positions of the tracked feature at different times are indicated in the figure. Panel (e) shows the bright spot's mean transverse positions with error bars during the propagation of the wave. We can see that the position
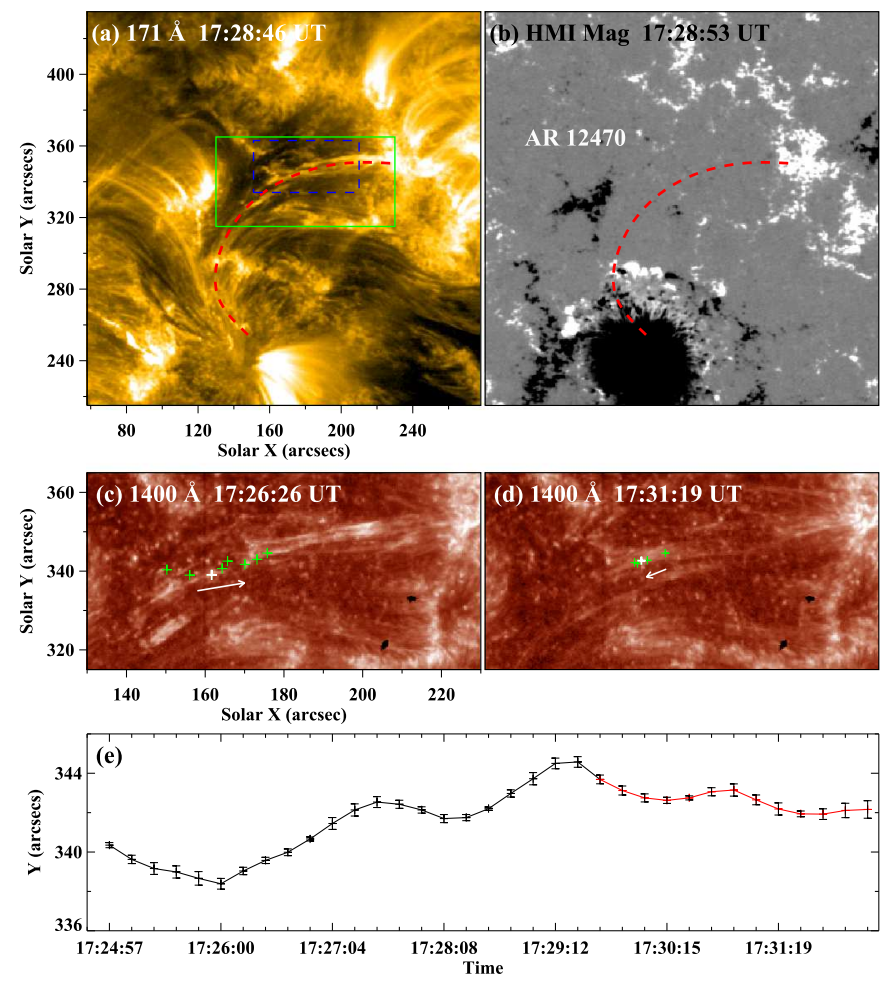

Fig. 3. Panels $a-b$ : SDO/AIA $171 \AA$ A image and SDO/HMI LOS magnetogram showing the EUV loops of the focused region on 2015 December 19 and its underneath magnetic fields. The red dashed lines delineate the magnetic loops along which the wave propagates. The green solid rectangle in panel $a$ outlines the FOV of panels $c-d$ while the blue dashed rectangle represents the FOV of $1400 \AA$ and $171 \AA$ A images in Figs. 4 and 5. Panels $c-d$ : IRIS SJIs of $1400 \AA$ displaying the propagation of the wave. The white arrows denote the propagating directions of the wave by tracking a bright spot. The green pluses outline the trajectory of the bright spot from 17:24:57 UT to 17:32:23 UT and the white pluses mask the positions of the spot at the moments shown in panels $c$ and $d$. Panel $e$ : the evolution of the spot's transverse position during the propagation of the wave. The black curve shows the wave propagating to the right (panel c) while the red to the left (panel d). An animation of the $1400 \AA$ images is available online.

curve reveals a periodic oscillation, with an average period of $\sim 128 \mathrm{~s}$ and amplitude of $880 \pm 170 \mathrm{~km}$.

Also accompanying the second rotation, several bright loops tracked by the bright material display regular transverse oscillation. To investigate the transverse oscillation of these loops, we tracked a given loop from 17:21:58 UT to 17:31:19 UT. A bright spot inside this oscillating loop was tracked in a limited longitudinal range $\left(15^{\prime \prime}\right)$. We determined the coordinates of this spot at each frame in $1400 \AA$ passband with the same method used in Fig. 3. We then got the average values of the spot coordinates at different frames and their standard deviations. Figure 4 shows the mean transverse oscillation in $1400 \AA$ images (see animation). The transverse position trajectory of the tracking loop is outlined in panels (a)-(d), and panel (e) shows the change of the oscillating loop's transverse position versus time with the error bars. It is shown that the transverse oscillation is visible for approximately four periods, with obvious damping - the amplitude decreases from $1370 \pm 210 \mathrm{~km}$ to $690 \pm 140 \mathrm{~km}$. We counted the oscillation period as $\sim 143 \mathrm{~s}$, mean upward velocity as $28 \pm 5 \mathrm{~km} \mathrm{~s}^{-1}$, and mean downward velocity as $32 \pm 4 \mathrm{~km} \mathrm{~s}^{-1}$. Under the reminder of the IRIS observations, we noticed that the oscillation motion could be detected in a higher temperature. We applied the $171 \AA$ 

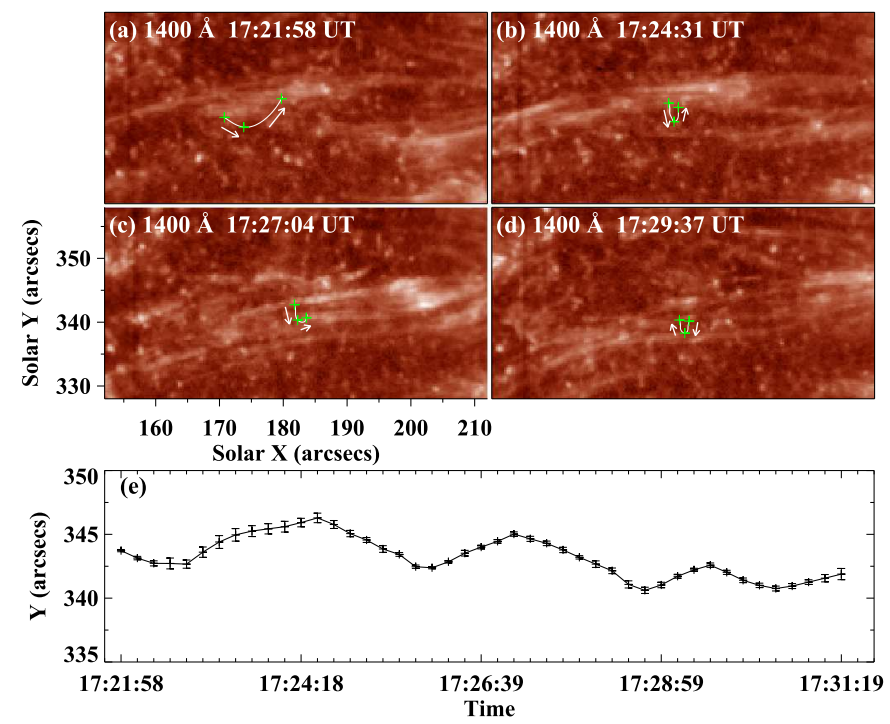

Fig. 4. Panels $a-d$ : sequence of IRIS $1400 \AA$ Amages showing the development of the transverse oscillation from 17:21:58 UT to 17:31:19 UT. The green pluses and white lines approximate the track of the loop's oscillation, and the white arrows indicate the moving directions of the tracked material. The FOV of these panels has been outlined by the blue dashed rectangle in Fig. 3a. Panel $e$ : the evolution of the tracking loop's transverse position during the oscillation. The full temporal evolution of the oscillation in $1400 \AA$ is available as an online animation.
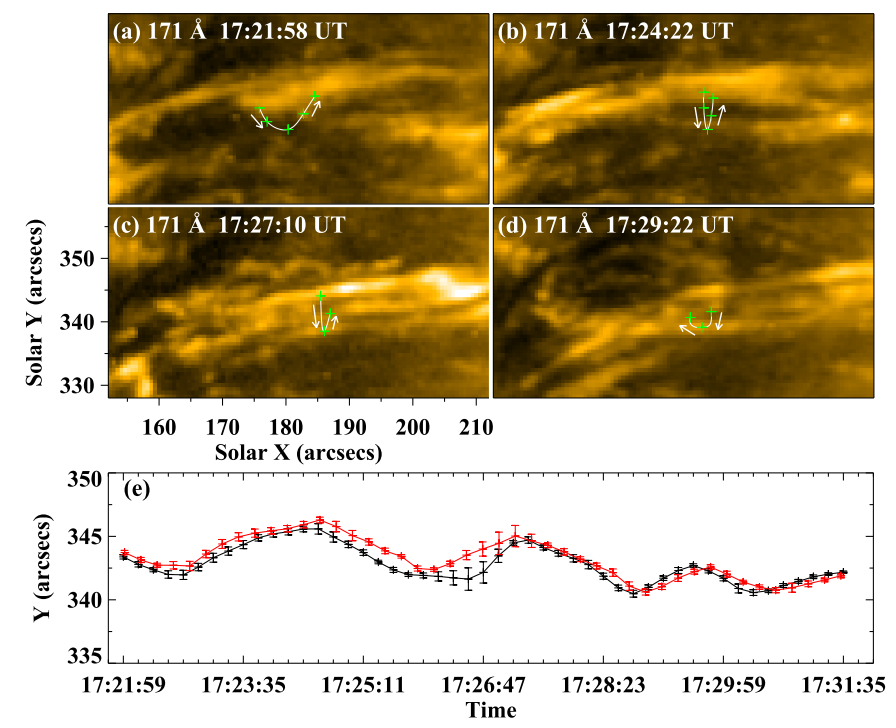

Fig. 5. Similar to Fig. 4, but in a higher temperature of the $171 \AA$ channel. The red curve are the duplications of Fig. $4 \mathrm{e}$.

data (see Fig. 5) and analyzed them in the same way as for the $1400 \AA$ channel. The oscillation is visible also for four periods, and the oscillation parameters, such as period, amplitude, and velocity, are nearly the same as those in the $1400 \AA$ bandpass (see Fig. 5e). We fitted the temporal evolution of the oscillation with a damped sine function described in Nakariakov et al. (1999) and obtained the decay time of $\sim 500 \mathrm{~s}$.

\section{Conclusions and discussion}

Employing the high-resolution observations from the IRIS and the SDO, we report a propagating wave in active region loops, which is triggered by filament activation. On 2015 December
19, a filament lay on the PIL of the NOAA AR 12470, which was activated and caused a C1.1 flare. Between the flare ribbons, a set of bright loops were detected to first rotate anticlockwise and then rotate clockwise. Following the end of the clockwise rotation, a propagating wave and an associated transverse oscillation were detected in the loops. In the $1400 \AA$ channel, there was bright material moving toward the west along the loops with a mean velocity of $66.2 \pm 1.1 \mathrm{~km} \mathrm{~s}^{-1}$ at first. Then, some bright material moved back to the east with a mean velocity of $12.7 \pm 1.3 \mathrm{~km} \mathrm{~s}^{-1}$. To investigate the transverse oscillation of these loops, we tracked a given loop and determined the transverse positions of the tracking loop in a limited longitudinal range $\left(15^{\prime \prime}\right)$. In both of $1400 \AA$ and $171 \AA$ channels, about four periods are distinguished during the transverse oscillation. The period of the oscillation is estimated as $\sim 143 \mathrm{~s}$, but the amplitude decreases from $1370 \pm 210 \mathrm{~km}$ to $690 \pm 140 \mathrm{~km}$.

In the chromosphere, only a few examples have been reported of waves or oscillations detected in fine structures over the disk. High resolution $\mathrm{H} \alpha$ filtergrams obtained with the Swedish Solar Telescope resolved evidence for small amplitude $\left(1-2 \mathrm{~km} \mathrm{~s}^{-1}\right.$ ) waves propagating along a number of on-disk filament threads. The oscillatory period of individual threads vary from three to nine minutes (Lin et al. 2007). Observations of Ca II $8542 \AA$ revealed a kink wave in an on-disk chromospheric active region fibril, and the properties of the wave were similar to those observed in off-limb spicules (Pietarila et al. 2011). Kanoh et al. (2016) employed simultaneous Hinode (Kosugi et al. 2007) and IRIS observations of a sunspot umbra to derive the upward energy flux at photosphere and lower transition region and estimate the energy dissipation. Temporal evolutions of the Doppler velocity, the magnetic flux density, and the line core intensity derived from Hinode/SP data were applied to determine the periodic oscillating features at photosphere. Moreover, the Doppler velocities derived from IRIS Mg II K and Si IV lines were used to detect the oscillations at the chromosphere and the lower transition region. They concluded that standing slow-mode waves are dominant at the photosphere, and the high-frequency leakage of the slow-mode waves is observed as upward waves at the chromosphere and the lower transition region. Bryans et al. (2016) reported strong recurring upward propagating flows observed by SDO/AIA with apparent speeds of $100-120 \mathrm{~km} \mathrm{~s}^{-1}$ at coronal loop footpoints. Furthermore, magnetoacoustic shock waves were detected by IRIS Mg IIh line. These observations illustrated that both shock waves and flows are involved at the footpoints. Different from previous works, here we first report a propagating wave with an associated transverse oscillation in active region loops, which is triggered by filament activation. In SDO/AIA $171 \AA$ and IRIS $1400 \AA$ channels, there is bright material moving along the loops with a wave-like shape. Meanwhile, the loops display transverse oscillation.

Before the detection of the propagating wave, we observed two rotation motions in the bright loops. The first rotation appeared between the two flare ribbons just at the peak of the flare around 17:10 UT. In the $1400 \AA$ observation, the bright material in the rotating loops is rotated anticlockwise if one looks from left to right along the rotation axis. To check this anticlockwise rotation, we analyzed the Si IV $1402.77 \AA$ line spectra along the slit which crosses the rotating loops. The spectral profiles in positions where the rotating loops cross the slit are approximated by single-Gaussian fits. Here we can imagine that there existed a tube, and the bright loops wound round this tube. Since the Doppler velocity in the lower position (blue plus) of the slit is $-24.9 \pm 0.9 \mathrm{~km} \mathrm{~s}^{-1}$ (blueshift) while that in upper 
position (red plus) is $31.2 \pm 0.5 \mathrm{~km} \mathrm{~s}^{-1}$ (redshift), we suggest that the red plus is located in upper side of the tube while the blue plus is located in the lower side and this rotation is anticlockwise when looking from left to right. Subsequently, the loops moved northward and reached the northernmost point around 17:21 UT. They then performed the second rotation motion - clockwise when looking form left to right along the rotation axis. Similar to the first rotation, we chose two positions along the slit for spectral analysis. The Doppler velocity in the upper position of the slit is $11.9 \pm 1.8 \mathrm{~km} \mathrm{~s}^{-1}$ (redshift) and that in lower position is $53.4 \pm 1.5 \mathrm{~km} \mathrm{~s}^{-1}$ (redshift). The same signal of Doppler velocity means that these two positions are located in the same side of this tube. Combing the fact that the redshift velocity of the lower position is bigger than that in the upper position, we propose that both of the two positions are located in the lower side of the tube and this rotation is clockwise when looking from left to right. Since the two rotation motions of these loops were in opposite directions, combining the fact that the first rotation appeared just when the flare peaked and the second rotation resulted in the appearance of a propagating wave, we propose that the energy released by the flare twisted the bright loops and was stored as non-potential magnetic energy in the twisted loops during the first rotation motion. Then in the second rotation, the stored energy was released by the unwinding motion of the twisted magnetic loops (Yan et al. 2014), leading to a transverse wave propagating along these loops.

Waves and oscillations can be detected either by imaging or spectroscopic observations in solar limb (Papushev \& Salakhutdinov 1994; Zaqarashvili et al. 2007; De Pontieu et al. 2007; He et al. 2009). On the solar disk, quasi-periodic propagating disturbances have been found in large coronal loops (e.g., Nakariakov et al. 1999; Nightingale et al. 1999; Berghmans \& Clette 1999; Aschwanden 2009; Ruderman \& Erdélyi 2009). The propagation of kink waves may lead to the observed periodic transverse displacement of the loop axis. When the velocity of the kink wave is polarized in the plane of observation, the spectral observation shows obvious Doppler shift in the location of oscillating loop. But if the velocity is polarized in the perpendicular plane, the visible displacement of the loop could be detected by the imaging observation. Since the observations in our study display propagating displacements of the active region loops in the $1400 \AA$ and $171 \AA$ channels, we interpret these oscillations as a propagating kink wave. This explanation is consistent with the reports of low amplitude kink oscillations in the solar corona (Tomczyk et al. 2007; Van Doorsselaere et al. 2008b). To estimate the density of the magnetic field in a fundamental kink oscillating loop, we applied the formula $B=\frac{2 L}{P} \sqrt{\mu m_{\mathrm{p}} m_{\mathrm{m}}\left(n_{\mathrm{in}}+n_{\mathrm{ex}}\right) / 2}$ (Roberts et al. 1984; Aschwanden $\&$ Schrijver 2011). The $P$ is the period of oscillation, $L$ is the length of the loop, $\mu$ is the magnetic permeability, $m_{\mathrm{m}}$ is the molecular weight, $m_{\mathrm{p}}$ is the proton mass, $n_{\mathrm{in}}$ and $n_{\mathrm{ex}}$ are the number densities of the proton inside and outside of the loop. We estimate that $L \sim 100 \mathrm{Mm}, P \sim 143 \mathrm{~s}, \mu=4 \pi, m_{\mathrm{m}}=1.2$ (Verwichte et al. 2013), $m_{\mathrm{p}} \sim 1.673 \times 10^{-24} \mathrm{~g},\left(n_{\text {in }}+n_{\mathrm{ex}}\right) / 2 \sim$ $9 \times 10^{8} \mathrm{~cm}^{-3}$ (Van Doorsselaere et al. 2008b). We then measured the magnitude of the magnetic field in the oscillating loop to be $\sim 21 \mathrm{G}$.

The transverse oscillation presented in this work was observed with a decay time of about $500 \mathrm{~s}$, comparable to previous results (Nakariakov et al. 1999; Wang \& Solanki 2004; White $\&$ Verwichte 2012). The decay of the loop oscillation amplitude indicates the dissipation of the wave energy, which contributes to the heating of the loops. To estimate the energy flux of the wave, we applied the formula $E_{\mathrm{w}}=\rho(v)^{2} V_{\mathrm{A}}$, where $\rho$ is the mass density, $v$ is the velocity amplitude, and $V_{\mathrm{A}}$ is the Alfvén speed (Tomczyk et al. 2007). The mass density was estimated to be $\rho=m_{\mathrm{p}} m_{\mathrm{m}}\left(n_{\mathrm{in}}+n_{\mathrm{ex}}\right) / 2 \sim 1.8 \times 10^{-15} \mathrm{~g} \mathrm{~cm}^{-3}$ (Van Doorsselaere et al. 2008b; Verwichte et al. 2013). We measured that the maximum displacement amplitude $\left(A_{\max }\right)$ is $\sim 1370 \mathrm{~km}$ and the oscillation period $(P)$ has a mean value of $\sim 143 \mathrm{~s}$. Then the maximum velocity amplitude $\left(v_{\max }\right)$ is estimated to be $\sim 60 \mathrm{~km} \mathrm{~s}^{-1}$ by the formula $v_{\max }=2 \pi A_{\max } / P$ (Ofman \& Wang 2008). Assuming the oscillation is a fundamental kink mode, we obtained the kink speed $C_{\mathrm{k}}=2 L / P \sim 1400 \mathrm{~km} \mathrm{~s}^{-1}$ and the Alfvén speed is $V_{\mathrm{A}}=C_{\mathrm{k}} / \sqrt{2} \sim 990 \mathrm{~km} \mathrm{~s}^{-1}$. Then we obtained the energy flux of the wave $E_{\mathrm{w}}=\rho(v)^{2} V_{\mathrm{A}} \sim 6.43 \times 10^{6} \mathrm{erg} \mathrm{cm}^{-2} \mathrm{~s}^{-1}$ with the $v$ taken as the maximum velocity amplitude $\left(v_{\max }\right)$. The estimated energy can balance typical radiative and conductive losses of the loops and heat them to coronal temperature (Withbroe \& Noyes 1977; Ofman \& Wang 2008). However, the event reported here is a special event triggered by a flare associated with filament activation. As a result, the mechanism in this event may not be responsible for heating of all the coronal loops.

Acknowledgements. We thank the referee for his/her valuable suggestions. The data are used courtesy of IRIS and SDO science teams. IRIS is a NASA small explorer mission developed and operated by LMSAL with mission operations executed at NASA Ames Research center and major contributions to downlink communications funded by ESA and the Norwegian Space Centre. This work is supported by the National Natural Science Foundations of China (11533008, 11790304, 11773039, 11673035, 11673034, and 11790300) and Key Programs of the Chinese Academy of Sciences (QYZDJ-SSW-SLH050).

\section{References}

Alfvén, H. 1947, MNRAS, 107, 211

Antolin, P., De Moortel, I., Van Doorsselaere, T., \& Yokoyama, T. 2017, ApJ, 836, 219

Aschwanden, M. J. 2009, Space Sci. Rev., 149, 31

Aschwanden, M. J., Schrijver, C. J. 2011, ApJ, 736, 102

Berghmans, D., Clette, F. 1999, Sol. Phys., 186, 207

Bradshaw, S. J., Klimchuk, J. A. 2015, ApJ, 811, 129

Bryans, P., McIntosh, S. W., De Moortel, I., \& De Pontieu, B. 2016, ApJ, 829, L18

De Pontieu, B., McIntosh, S. W., Carlsson, M., et al. 2007, Science, 318, 1574

De Pontieu, B., Title, A. M., Lemen, J. R., et al. 2014, Sol. Phys., 289, 2733

Erdélyi, R., \& Fedun, V. 2007, Science, 318, 1572

Guo, Y., Erdélyi, R., Srivastava, A. K., et al. 2015, ApJ, 799, 151

He, J.-S., Tu, C.-Y., Marsch, E., et al. 2009, A\&A, 497, 525

Jafarzadeh, S., Solanki, S. K., Gafeira, R., et al. 2017, ApJS, 229, 9

Kanoh, R., Shimizu, T., \& Imada, S. 2016, ApJ, 831, 24

Klimchuk, J. A. 2006, Sol. Phys., 234, 41

Kosugi, T., Matsuzaki, K., Sakao, T., et al. 2007, Sol. Phys., 243, 3

Kuridze, D., Morton, R. J., Erdélyi, R., et al. 2012, ApJ, 750, 51

Kuridze, D., Verth, G., Mathioudakis, M., et al. 2013, ApJ, 779, 82

Lemen, J. R., Title, A. M., Akin, D. J., et al. 2012, Sol. Phys., 275, 17

Li, H., Liu, Y., \& Vai Tam, K. 2017, ApJ, 842, 99

Li, L. P., Peter, H., Chen, F., \& Zhang, J. 2014, A\&A, 570, A93

Li, T., \& Zhang, J. 2012, ApJ, 760, L10

Li, T., Yang, K., Hou, Y., \& Zhang, J. 2016, ApJ, 830, 152

Lin, Y., Engvold, O., Rouppe van der Voort, L. H. M., \& van Noort, M. 2007, Sol. Phys., 246, 65

Nakariakov, V. M., Ofman, L., Deluca, E. E., Roberts, B., \& Davila, J. M. 1999, Science, 285,862

Nightingale, R. W., Aschwanden, M. J., \& Hurlburt, N. E. 1999, Sol. Phys., 190, 249

O’Dwyer, B., Del Zanna, G., Mason, H. E., Weber, M. A., \& Tripathi, D. 2010, A\&A, 521, A21

Ofman, L., \& Wang, T. J. 2008, A\&A, 482, L9

Okamoto, T. J., \& De Pontieu, B. 2011, ApJ, 736, L24

Okamoto, T. J., Antolin, P., De Pontieu, B., et al. 2015, ApJ, 809, 71

Oliver, R., Ruderman, M. S., \& Terradas, J. 2014, ApJ, 789, 48

Papushev, P. G., \& Salakhutdinov, R. T. 1994, Space Sci. Rev., 70, 47

Parker, E. N. 1958, ApJ, 128, 664 
Parker, E. N. 1988, ApJ, 330, 474

Pereira, T. M. D., Rouppe van der Voort, L., \& Carlsson, M. 2016, ApJ, 824, 65

Pesnell, W. D., Thompson, B. J., \& Chamberlin, P. C. 2012, Sol. Phys., 275, 3

Peter, H., Tian, H., Curdt, W., et al. 2014, Science, 346, 1255726

Pietarila, A., Aznar Cuadrado, R., Hirzberger, J., \& Solanki, S. K. 2011, ApJ, 739, 92

Roberts, B., Edwin, P. M., \& Benz, A. O. 1984, ApJ, 279, 857

Ruderman, M. S., \& Erdélyi, R. 2009, Space Sci. Rev., 149, 199

Schou, J., Scherrer, P. H., Bush, R. I., et al. 2012, Sol. Phys., 275, 229

Shen, Y., Liu, Y. D., Chen, P. F., \& Ichimoto, K. 2014, ApJ, 795, 130

Solanki, S. K., Lagg, A., Woch, J., Krupp, N., \& Collados, M. 2003, Nature, 425, 692

Terradas, J., Arregui, I., Oliver, R., \& Ballester, J. L. 2008, ApJ, 678, L153

Tian, H., McIntosh, S. W., Wang, T., et al. 2012, ApJ, 759, 144

Tian, H., DeLuca, E., Reeves, K. K., et al. 2014, ApJ, 786, 137
Tomczyk, S., McIntosh, S. W., Keil, S. L., et al. 2007, Science, 317, 1192

Van Doorsselaere, T., Nakariakov, V. M., \& Verwichte, E. 2008a, ApJ, 676, L73

Van Doorsselaere, T., Nakariakov, V. M., Young, P. R., \& Verwichte, E. 2008b, A\&A, 487, L17

Verwichte, E., Van Doorsselaere, T., Foullon, C., \& White, R. S. 2013, ApJ, 767, 16

Wang, T. J., \& Solanki, S. K. 2004, A\&A, 421, L33

White, R. S., \& Verwichte, E. 2012, A\&A, 537, A49

Withbroe, G. L., \& Noyes, R. W. 1977, ARA\&A, 15, 363

Wright, P. J., Hannah, I. G., Grefenstette, B. W., et al. 2017, ApJ, 844, 132

Yan, X. L., Xue, Z. K., Liu, J. H., Kong, D. F., \& Xu, C. L. 2014, ApJ, 797, 52

Zaqarashvili, T. V., Khutsishvili, E., Kukhianidze, V., \& Ramishvili, G. 2007, A\&A, 474, 627

Zaqarashvili, T. V., \& Erdélyi, R. 2009, Space Sci. Rev., 149, 355 